\title{
Rest and Therapeutic Interventions Following Sport-Related Concussion: An Evidence-to-Practice Review
}

Alyssa L. Bolno, SCAT, ATC*; Brittni G. Hoover, MS, ATC*; Ryan J. Michels, SCAT, ATC*; Allison B. Smith, PhD, ATC + ; Zachary K. Winkelmann, PhD, SCAT, ATC*

*University of South Carolina, Columbia, SC; †University of Louisiana - Lafayette, Lafayette, LA

\begin{abstract}
Sport-related concussion (SRC) is among the most common injuries seen in both sports and recreation, especially collision and full contact sports. However, there has been controversy surrounding which recovery protocol would be best used in the rehabilitation of a person who has suffered from SRC. Symptoms and dysfunctions can vary from patient to patient. The average recovery time for adults is 14 days post incidence. Although most patients recover over the first few days/weeks post-injury, $15 \%$ reported recovery time of 30 or more days. Previous research determined a need for cognitive and physical rest of 24-48 hours, or until acute symptoms resolve to then begin a six-step progressive returnto-activity. Evidence from recent research shows that prolonged physical and cognitive rest may not be as effective as previously determined for concussion and postconcussion syndrome management. Multiple studies have shown that a multimodal active recovery helps patients diagnosed with concussion or post-concussion syndrome have a timely return-to-activity, as well as increases improvement in overall symptoms. Within these studies, researchers have determined that patients should avoid exacerbating symptoms by staying below their symptom threshold, which can be determined through the implementation of the Buffalo Concussion Treadmill Test. Although not all research has shown an increase in recovery from SRC using active rehabilitation, a substantial number of researchers have seen a positive effect. Even with the limited amount of research and evidence, clinicians should highly consider the use of active rehabilitation following a 24 to 48 hour period of rest for SRC. Therefore, primary purpose of this article is to evaluate the evidence related to determining if rest is beneficial following a concussion, and what the optimal duration of rest should be. The secondary purpose is to evaluate the evidence of active treatment, and rehabilitation effectiveness, for athletes who have experienced SRC.

Key Phrases

Therapeutic exercise; functional testing
\end{abstract}

\section{Correspondence}

Dr. Zachary Winkelmann, 1300 Wheat Street, Columbia, SC 29208.

E-mail:winkelz@mailbox.sc.edu

Twitter: @zachwinkelmann

Full Citation

Bolno AL, Hoover BG, Michels RJ, Smth AB, Winkelmann ZK. Rest and therapeutic interventions following sport-related concussion: An evidence-topractice review. Clin Pract Athl Train. 2021 ;4(3): 10 16. https://doi.org/10.31622/2021/0004.3.3.

Submitted: December 3, 2020 Accepted: May 6, 2021.

Copyright (C) by Indiana State University All rights reserved. ISSN Online 2577-8188

\section{ORIGINAL REFERENCE}

Schneider KJ, Leddy JJ, Guskiewicz KM, Seifert $T$, McCrea $M$, Silverberg ND, FeddermannDemont N, Iverson GL, Hayden A, Makdissi M. Rest and treatment/rehabilitation following sport-related concussion: a systematic review. $\mathrm{Br} J$ Sports Med. 2017;51(12):930-934. https://doi.org10.1136/bisports-2016097475.

\section{SUMMARY}

\section{CLINICAL PROBLEM AND QUESTION}

Sport-related concussion (SRC) is among the most common injuries seen in sports and recreation, especially in collision and full contact sports and has been defined as external or biomechanical force causing neurological and altered brain function impairments along functional disturbances leading to clinical signs and symptoms., ${ }^{1,2}$ Sportrelated concussion has a heterogeneous clinical presentation representing multiple subtypes of disease. At the time of suspected SRC, a multifaceted assessment starts with immediate considerations such as cervical spine injury and neurological assessment followed by extensive concussion assessment including orientation, memory, cognition, balance, and vestibular oculomotor screening (VOMS) completed by a licensed healthcare provider with training and education in sport-related concussion management. $^{2}$ Symptoms and neurological problems following a concussion can vary from patient to patient with typical recovery time for adults being reported around 14 days.1,3 Although most patients recover over the first 10 14 days following the injury, $15 \%$ have reported trajectory of 30 days or more. ${ }^{3}$ As of 2019, 
rehabilitation guidelines are based on expert opinion found in a consensus documents based on group meetings from concussion experts including researchers in clinical medicine, sports medicine, neuroscience, neuroimaging, athletic training and sports science. 2,4 These individuals concluded a need for a period of cognitive and physical rest of 24-48 hour, or until acute symptoms resolve, followed by a progressive six-step return to academics and sport, is the best course of action. ${ }^{2}$ This period of rest is critical due to the pathophysiological changes in the brain during the first 48 hours such as: ion influx leading to migraines and headaches; a decrease in glucose metabolism and metabolite $\mathrm{N}$-acetylaspartate (NAA) in frontal white matter leading to increased risk of second injury; and finally impaired neurotransmission leading to impaired cognition, slowed processing, and slowed reaction time. ${ }^{5}$ Due to this critical period being recommended, the primary purpose of this article was to evaluate the evidence related to determining if rest is beneficial following a concussion and what the optimal duration of rest (after the 48-hour period) should be. The secondary purpose of this article was to evaluate the evidence of active treatment and rehabilitation effectiveness for athletes who have experienced SRC. ${ }^{3}$

\section{SUMMARY OF LITERATURE}

The initial search terms selected for the search strategy were created and modified by the original authors of the systematic review. The following electronic databases were used for the search strategy: MEDLINE, CINAHL, PsycINFO, Cochrane Central Register of Controlled Trials, SPORTDiscus, EMBASE and Proquest Dissertations and Theses Global. ${ }^{3}$ Inclusion criteria for the review included (1) original research, (2) the focus of the study had SRC as a source of injury, and (3) the study evaluated the effect of rest or treatment. $^{2}$ The Downs and Black (DB) checklist was used to evaluate the risk of bias in the articles and it was used in cases when the authors did not

Copyright (C) by Indiana State University All rights reserved. ISSN Online 2577-8188 agree on a decision. A total of 28 studies with 3218 participants were included in the systematic review, which included five randomized control trials, nine cohort studies, two quasi-experimental studies, and 12 case studies. ${ }^{3}$

\section{SUMMARY OF OUTCOMES}

There were various interventions and treatment options listed within the nineteen studies. Research determined strict rest after SRC decreases energy and patients had longer symptom period of more than 10-14 day average. ${ }^{2}$ However, there is conflicting data that shows the efficiency of rest following a concussion that aids the recovery of function and symptom resolution, Studies that researched treatments for concussion were categorized into rehabilitation, exercise, medical and other (symptom-free waiting period). Rehabilitation treatment was divided into multimodal physiotherapy, collaborative care, cognitive rehabilitation, and multifaceted physical therapy with exercise. Multifaceted rehabilitation included treatment regimens such as adaptation, balance, musculoskeletal, aerobic, anaerobic, and sport specific. ${ }^{3}$ Other areas within rehabilitation that were mentioned were vestibular and manual therapy, specifically exercises that focus on improving pain and function for patients with cervical spine symptoms. Vestibular therapies were reported to have a positive effect on symptoms and recovery, such as improving neck pain, headaches and dizziness. Active recovery treatment involved exercise which introduced subsymptom submaximal aerobic exercise multiple times per week, which did not exacerbate symptoms but rather improved symptom scores and the time for return to baseline cognitive scores.

Medical treatment was divided into either interventional or traditional pharmacological measures to help with post-traumatic headaches. ${ }^{3}$ Treatment in these categories only showed minimal benefits. All these interventions were 
compared against rest and a symptom-free waiting period to determine which intervention helped decrease SRC symptoms and facilitate a safe and timely return-to-activity.

\section{FINDINGS AND CLINICAL IMPLICATIONS}

The guiding systematic review compared articles that were focused on concussion research that analyzed the overall effects of rehabilitation, medical, and other forms of post-concussion treatment on patients. ${ }^{3}$ Individuals who experience concussions can experience neurological, vestibular, oculomotor, along with physical ailments due to the etiology of the injury. Of these treatment types, none were found to have better outcomes over the other. As a result of these findings, additional research may be required on these treatments. However, there is data that suggest that post-concussion patients can begin to partake in physical and cognitive functions after 24 to 48 hours of rest. ${ }^{3}$ Manual therapy, such as cervical lateral glides and occipital muscle energy, combined with specific exercises involving either balance, adaptation, or low level aerobics were found to improve function and pain in patients with SRC. ${ }^{2}$ Clinicians need to understand the importance of completing these tasks in which the individual stays under the symptom exacerbation threshold to avoid increasing symptoms, ${ }^{3}$ which can be done by implementing the Buffalo Concussion Treadmill Test (BCTT) as demonstrated in Figure 1. By using this test, clinicians can determine the exact level of aerobic activity and heart rate that exacerbates symptoms. The completion of the BCTT can correlate to the duration of clinical recovery as well as give an estimate on the stage of return-toplay (RTP) process and to help understand how much rehabilitation exercise to prescribe within each treatment session for the patient. 6

Regardless of the treatment chosen, it is important for both the clinician and patient to keep the symptoms underneath the exacerbation during

Copyright (C) by Indiana State University All rights reserved. ISSN Online 2577-8188 post-concussion assessment. ${ }^{3}$ From the research conducted, indications for rehabilitation programs involving a controlled symptom threshold limit, with submaximal exercise for athletes who have persistent symptoms, may be of long-term benefit. $^{3}$ Clinicians should return athletes gradually to their non-sport activities, as tolerated while making sure the patient does not have any return of symptoms 24 hours before progressing in the process, and should avoid heavy exertion related activities until fully cleared.1,7,8 Specifically, the 2016 Berlin Consensus Statement, recommended a five stage return-to-learn ( $R T L$ ) protocol in which children and adolescents should gradually resume academics before returning to sport or other physical activity. The consensus statement did agree that while gradually returning the athlete to school, the patient could begin an early introduction into symptoms limited activity, such as low cardiovascular exercises determined by BCTT, following the 24 to 48 hours rest period. ${ }^{1,8}$ The RTL process is important due to the changes in attention, cognitive processing speed, short term memory and executive function that makes learning difficult following an SRC. Clinicians should introduce the RTL prior to RTP as integration of learning may exacerbate neurological changes which can in return affect the overall RTP process. ${ }^{8}$

In terms of specific rehabilitation options, vestibular rehabilitation was found to show positive effects, especially with patients who have persistent vestibular findings after SRC. ${ }^{3}$ Examples of vestibular rehabilitation used include Cawthorne-Cooksey exercises, sensory integration balance training, gaze stabilization, ocular tracking, vestibular ocular reflex training, and dynamic gate. Other technology that can be used in vestibular rehabilitation includes Dynavision D2, Fit Light System, Geobard, and the Interactive Metronome Neurotiming. ${ }^{3}$ If clinicians are looking for confirmation of vestibular oculomotor dysfunction following a SRC, clinicians should look to use to VOMS, which has unique 
aspects for determining vestibular function not assessed by other post-concussion test. ${ }^{3}$ Before performing the VOMS, clinicians either need to be trained in administering the exam or refer to a healthcare professional who is trained in administering the exam for the most accurate results. ${ }^{9}$ There is little to no evidence to support the use of pharmacological management strategies such as analgesics, non-steroidal antiinflammatory drugs, or acetaminophen for treating SRC.3,10 Every individual is different and there is no standard timeline for how and when a patient can and will recover from a concussion. The return-to-activity decision should be decided by an interprofessional healthcare team, and all factors closely analyzed.

\section{CLINICAL BOTTOM LINE}

Evidence from recent reviews suggest that prolonged physical and cognitive rest may not be as effective as previously thought for concussion and post-concussion syndrome management. ${ }^{11}$ Multiple studies have shown that a multimodal active recovery, including progressive levels of exercise difficulty that increase throughout RTP, helps athletes diagnosed with concussion or postconcussion syndrome efficiently return-to-activity and helps with their overall symptoms and recovery time. ${ }^{12-14}$ One research study concluded that prolonged rest could impede athlete recovery and lead to mood and/or anxiety disorders. ${ }^{14}$ Grabowski et al. (2017) was able to further suggest supervised physical rehabilitation, involving sub-symptom cardiovascular exercise, vestibular/oculomotor therapy, and cervical spine rehabilitation, improved symptoms scores in exercise tolerance and on graded exercise testing and balance. 12 Other studies, such as Chrisman et al. (2017) who used the Balke treadmill test, which is similar to the BCTT, demonstrated symptoms decreased exponentially following a progressive aerobic exercise program regardless of symptoms duration at presentation and none of the subjects had worsening of symptoms throughout the program. ${ }^{13}$ Implementation of lowlevel activity including sub-symptom cardiovascular exercise including biking, walking, and jogging, are a few ways to avoid excessive rest following concussion. ${ }^{11}$ Overall, it is becoming clear that prolonged and sustained rest from all activity following a concussion is not beneficial and could potentially be harmful to the recovery process.

Previously, patients were instructed on complete rest from both physical and mental activity until all symptoms were no longer present. The recent research indicates a change in treatment for SRC to a more active approach after 24-to-48-hour rest, may aid in recovery. Although there is some conflicting evidence on active rehabilitation providing a benefit towards SRC recovery, it should still be considered due to its potential use in clinical practice. Clinicians should still follow the protocol of 24 to 48 hours of rest post SRC before they begin incorporating active rehabilitation. Vestibular rehabilitation such as: CawthorneCooksey exercises, sensory integration balance training, and vestibulo-ocular reflex, or cervical manual therapy, have shown the most positive effects in regards to overall symptom recovery and faster RTP of all the different concussion related interventions. ${ }^{3}$ The use of exercise tolerance in the active rehabilitation protocol can be useful for the differential diagnosis of patients with potential post-concussion syndrome and can also act as a physiological sign of readiness to RTA. ${ }^{11}$

If active rehabilitation is the chosen path of SRC treatment, the clinician should always be aware and ensure the patient's symptoms are staying under the original reported symptom scores after the initially recorded SRC. Symptom scores should be taken daily while going through the rehabilitation process to ensure the symptoms are resolving and not over the scores from the initially recorded SRC. ${ }^{6}$ Despite the inconclusive findings on the effectiveness for active rehabilitation in the 
Table 1: Key Information, Helpful Tips, and Take-Home Messages of Management of Sport-Related Concussion (SRC)

Key Information

SRC is among the most common injuries seen in sports and recreation, especially in collision and full contact sports. ${ }^{1}$ Typically, recovery time for adults has been reported as 14 days. ${ }^{1}$

- Symptoms decrease exponentially following a progressive aerobic exercise program, regardless of symptoms duration at presentation, and all of the subjects were without symptom exacerbation throughout the program. ${ }^{9}$

\section{Helpful Tips}

At the time of suspected SRC, a multifaceted assessment starts with ruling out considerations such as cervical spine injury and neurological assessment followed by extensive concussion assessment including orientation, memory, cognition, balance, and vestibular ocular motor screening. All should be completed by a credentialed medical professional who has specialized training in SRC diagnosis and management.

- An efficient way of ensuring the patient's symptoms stay below the limit of symptoms worsening would be to implement the Buffalo Concussion Treadmill Test. By using this test, you can find the exact level of aerobic activity and heart rate that exacerbates symptoms, which may also correlate to the duration of clinical recovery. ${ }^{3}$

\section{Take-Home Messages for Clinician}

Follow the guidance of the medical staff, in collaboration with utilizing evidence-based practice.

- Clinicians should return athletes gradually to their non-sport activities, as tolerated, and should avoid heavy exertion related activities until fully cleared. Activities allowed should be based on patient symptoms but can include light cardiovascular or vestibular exercises.

- Specifically, the Berlin Consensus Statement (2016) recommended a 5 stage return-to-learn protocol in which children and adolescents should gradually be returned to academics before RTP. ${ }^{4}$

- The return-to-play decision should be managed as a collaborative effort amongst healthcare providers such as athletic trainers, team physician and SRC specialist and all factors such as neurological function, symptom checklist, and performance on concussion assessment should be closely analyzed.

management of a SRC, athletic trainers should consider the use of the BCTT due to its ability to objectively measure aerobic exercise capacity. ${ }^{6}$ The BCTT can be used safely on either a treadmill or a stationary bike. While the paradigm of rest and symptom recovery has been well adopted in sports medicine for concussion management, the guiding systematic review identified positive effects in most of the studies (16/19) for postconcussion treatment. ${ }^{3}$ We recommend that athletic trainers and other sports medicine professionals managing SRC to evolve their practice away from complete rest and move towards an integration of active rehabilitation. Overall, we suggest that clinicians use active rehabilitation following a 24 to 48 hour period of rest for SRC. The management of a SRC is also complex and multifaceted meaning an integrated approach is necessary from multiple providers. As athletic trainers begin to reconsider the management process through a collaborative and

Copyright (C) by Indiana State University All rights reserved. ISSN Online 2577-8188 interprofessional lens, we suggest using Table 1 take home and key messages to guide your discussions. Following the discussions, we suggest the sports medicine team integrate a quality improvement process whereby small changes, including policy and implementation of active rehabilitation strategies, are undertaken segmentally to learn more about the necessary skills and resources for the changes to their care plan.

\section{REFERENCES}

1. McCrory P, Meeuwisse W, Dvořák J, et al. Consensus statement on concussion in sport-the 5 (th) international conference on concussion in sport held in Berlin, October 2016. Br J Sports Med. 2017;51(1 1):838-847. https://doi.org/10.1136/bjsports-2017097699.

2. Schneider KJ. Concussion part II: Rehabilitation - The need for a multifaceted Volume 4-Issue 3 - November 2021 
approach. Musculoskelet Sci Pract. 2019;42:151-161.

https://doi.org/10.1016/i.msksp.2019.01.0 06.

3. Schneider KJ, Leddy JJ, Guskiewicz KM, et al. Rest and treatment/rehabilitation following sport-related concussion: a systematic review. $\mathrm{Br} J$ Sports Med. 2017;51(12):930-934. https://doi.org/10.1136/bisports-2016097475.

4. McCrory $P$, Meeuwisse WH, Aubry $M$, et al. Consensus statement on concussion in sport: the 4th International Conference on Concussion in Sport held in Zurich, November 2012. J Am Coll Surg. 2013;216(5):e55-71. https://dx.doi.org/10.4085\%2F10626050-48.4.05.

5. Giza CC, Hovda DA. The new neurometabolic cascade of concussion. Neurosurgery. $2014 ; 75 \quad$ Suppl $4(0 \quad 4):$ S24-33. https://doi.org/10.1227/neu.0000000000 $\underline{000505 .}$.

6. Haider MN, Leddy JJ, Wilber CG, et al. The Predictive Capacity of the Buffalo Concussion Treadmill Test After Sport-Related Concussion in Adolescents. Front Neurol. 2019;10:395. https://doi.org/10.3389/fneur.2019.00395

7. Harmon KG, Drezner JA, Gammons M, et al. American Medical Society for Sports Medicine position statement: concussion in sport. Br J Sports Med. 2013;47(1):15-26. https://doi.org/10.1136/bjsports-2012091941.

8. Harmon KG, Clugston JR, Dec K, et al. American Medical Society for Sports Medicine position statement on concussion in sport. Br J Sports Med. 2019;53(4):213-225. https://doi.org/10.1136/bjsports-2018100338.

9. Yorke AM, Smith L, Babcock M, Alsalaheen B. Validity and Reliability of the Vestibular/Ocular Motor Screening and Associations With Common Concussion Screening Tools. Sports Health.

Copyright $(\subset$ by Indiana State University All rights reserved. ISSN Online 2577-8188
2017;9(2):174-180.

https://doi.org/10.1177/1941738116678 411.

10. Larsen EL, Ashina H, lliazi A, et al. Acute and preventive pharmacological treatment of post-traumatic headache: a systematic review. J Headache Pain. 2019;20(1):98. https://doi.org/10.1186/s10194-0191051-7.

11. Leddy JJ, Wilber CG, Willer BS. Active recovery from concussion. Curr Opin Neurol. 2018;31(6):681-686.

https://doi.org/10.1097/wc0.0000000000 000611.

12. Grabowski P, Wilson J, Walker A, Enz D, Wang $S$. Multimodal impairment-based physical therapy for the treatment of patients with post-concussion syndrome: A retrospective analysis on safety and feasibility. Phys Ther Sport. 2017;23:22-30. https://doi.org/10.1016/i.ptsp.2016.06.00 1.

13. Chrisman SPD, Whitlock KB, Somers E, et al. Pilot study of the Sub-Symptom Threshold Exercise Program (SSTEP) for persistent concussion symptoms in youth. NeuroRehabilitation. 2017;40(4):493-499. https://doi.org/10.3233/nre-161436.

14. Mahooti N. Sports-Related Concussion: Acute Management and Chronic Postconcussive Issues. Child Adolesc Psychiatr Clin N Am. 2018;27(1):93-108.

https://doi.org/10.1016/i.chc.2017.08.005. 
Figure 1: Buffalo Concussion Treadmill Test Protocol

Prior to Testing Start:

1) Inform the patient about test procedures and what to expect during the test

2) Explain and demonstrate the Borg Rating of Perceived Exertion (RPE) scale and obtain resting scores (see graphic representation below)

3) Remind patient that they will be asked to rate exertion and symptom severity at each minute during exercise

\begin{tabular}{|c|c|}
\hline $6-7$ & Very, very light \\
\hline $8-9$ & Very light \\
\hline $10-11$ & Fairly light \\
\hline $12-13$ & Somewhat hard \\
\hline $14-16$ & Hard \\
\hline $17-18$ & Very hard \\
\hline 19 & Very, very hard \\
\hline 20 & Max exertion \\
\hline
\end{tabular}

\begin{tabular}{|c|c|c|c|c|c|}
\hline $\begin{array}{l}\text { Patient stands on } \\
\text { end of treadmill } \\
\text { Height (in) of } \\
\text { patient is taken } \\
>5.5^{\prime \prime}=3.6 \mathrm{mph} \\
<5.5^{\prime \prime}=3.2 \mathrm{mph} \\
\text { Incline }=0^{\circ}\end{array}$ & $\begin{array}{l}\text { After each minute, } \\
\text { increase incline } \\
\text { by } 1^{\circ} \\
\text { Rate RPE and } \\
\text { symptom severity } \\
\text { or heart rate } \\
\text { *1-point is given for } \\
\text { any worsening } \\
\text { syptom, } 1 \text {-point is } \\
\text { given for any new } \\
\text { symptom }\end{array}$ & $\begin{array}{l}\text { Treadmill incline } \\
\text { increases at } \\
1 \% \text { minute } \\
\text { Athletic trainer } \\
\text { should note the } \\
\text { Likert scale } \\
\text { reason/symptom } \\
\text { that sparked } \\
\text { increase }\end{array}$ & $\begin{array}{l}\text { Maximum incline } \\
=15^{\circ} \text { or } 12^{\circ} \text { at } \\
\text { modified test } \\
\text { Speed increases } \\
\text { by } 0.4 \text { mph each } \\
\text { minute with no } \\
\text { incline change }\end{array}$ & $\begin{array}{l}\text { Terminate the test } \\
\text { when RPE is } \\
\text { reported at } 19.5 \\
\text { or once patient } \\
\text { reports inability } \\
\text { to continue due to } \\
\text { symptoms } \\
\text { *Max time on } \\
\text { treadmill: } 20 \\
\text { minutes }\end{array}$ & $\begin{array}{l}\text { Once test has } \\
\text { been terminated, } \\
\text { reduce speed to } \\
2.5 \text { mph and } \\
\text { incline reduced } \\
\text { back to } 0^{\circ} \text { for a } \\
2 \text { minute } \\
\text { cooldown } \\
\text { Patient should rest } \\
\text { until symptoms } \\
\text { return to pre- } \\
\text { BCTT value }\end{array}$ \\
\hline
\end{tabular}

Modified iteration of Schneider KJ, Leddy JJ, Guskiewicz KM, Seifert T, McCrea M, Silverberg ND, Feddermann-Demont N, Iverson GL, Hayden A, Makdissi M. Rest and treatment/rehabilitation following sport-related concussion: a systematic review. Br J Sports Med. 2017;51(1 2):930-934. doi: 10.1 136/bisports-2016-097475. 